\title{
Generalized extended Navier-Stokes theory \\ Correlations in molecular fluids with intrinsic angular momentum
}

Hansen, J. S.; Daivis, Peter J.; Dyre, Jeppe C.; Todd, B. D.; Bruus, Henrik

Published in:

Journal of Chemical Physics

Link to article, DOI:

$10.1063 / 1.4774095$

Publication date:

2013

Document Version

Publisher's PDF, also known as Version of record

Link back to DTU Orbit

Citation (APA):

Hansen, J. S., Daivis, P. J., Dyre, J. C., Todd, B. D., \& Bruus, H. (2013). Generalized extended Navier-Stokes theory: Correlations in molecular fluids with intrinsic angular momentum. Journal of Chemical Physics, 138(3), 034503. https://doi.org/10.1063/1.4774095

\section{General rights}

Copyright and moral rights for the publications made accessible in the public portal are retained by the authors and/or other copyright owners and it is a condition of accessing publications that users recognise and abide by the legal requirements associated with these rights.

- Users may download and print one copy of any publication from the public portal for the purpose of private study or research.

- You may not further distribute the material or use it for any profit-making activity or commercial gain

- You may freely distribute the URL identifying the publication in the public portal 


\section{AIP $\begin{gathered}\text { molowanat } \\ \text { chemical Physics }\end{gathered}$}

\section{Generalized extended Navier-Stokes theory: Correlations in molecular}

fluids with intrinsic angular momentum

J. S. Hansen, Peter J. Daivis, Jeppe C. Dyre, B. D. Todd, and Henrik Bruus

Citation: J. Chem. Phys. 138, 034503 (2013); doi: 10.1063/1.4774095

View online: http://dx.doi.org/10.1063/1.4774095

View Table of Contents: http://jcp.aip.org/resource/1/JCPSA6/v138/i3

Published by the American Institute of Physics.

\section{Additional information on J. Chem. Phys.}

Journal Homepage: http://jcp.aip.org/

Journal Information: http://jcp.aip.org/about/about_the_journal

Top downloads: http://jcp.aip.org/features/most_downloaded

Information for Authors: http://jcp.aip.org/authors

\section{ADVERTISEMENT}

\section{Instruments for advanced science}
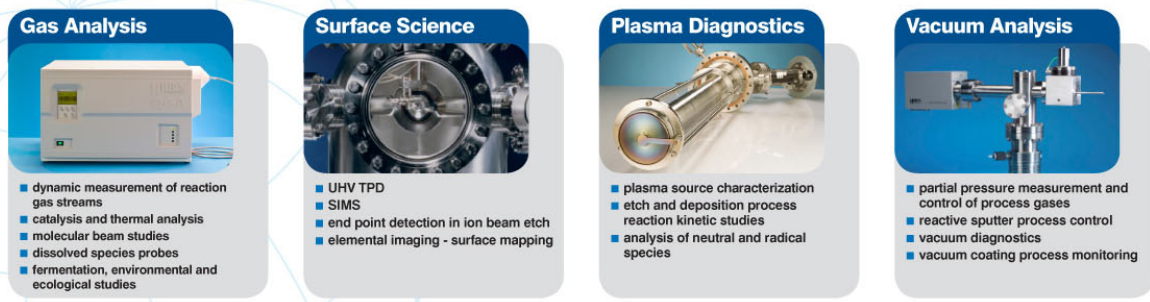

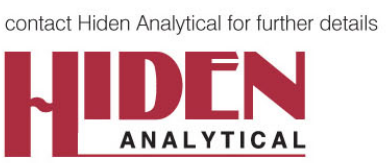

info@hideninc.com www.HidenAnalytical.com CLICK to view our product catalogue 


\title{
Generalized extended Navier-Stokes theory: Correlations in molecular fluids with intrinsic angular momentum
}

\author{
J. S. Hansen, ${ }^{1, a)}$ Peter J. Daivis, ${ }^{2}$ Jeppe C. Dyre, ${ }^{1}$ B. D. Todd, ${ }^{3}$ and Henrik Bruus ${ }^{4}$ \\ ${ }^{1}$ DNRF Centre "Glass and Time," IMFUFA, Department of Sciences, Roskilde University, Postbox 260, \\ DK-4000 Roskilde, Denmark \\ ${ }^{2}$ Applied Physics, School of Applied Sciences, RMIT University, GPO Box 2476, Melbourne, \\ Victoria 3001, Australia \\ ${ }^{3}$ Mathematics Discipline, Faculty of Engineering and Industrial Sciences, and Center for Molecular \\ Simulation, Swinburne University of Technology, P.O. Box 218, Hawthorn, Victoria 3122, Australia \\ ${ }^{4}$ Department of Physics, Technical University of Denmark, DTU Physics Building 309, DK-2800 Kongens \\ Lyngby, Denmark
}

(Received 6 September 2012; accepted 18 December 2012; published online 16 January 2013)

\begin{abstract}
The extended Navier-Stokes theory accounts for the coupling between the translational and rotational molecular degrees of freedom. In this paper, we generalize this theory to non-zero frequencies and wavevectors, which enables a new study of spatio-temporal correlation phenomena present in molecular fluids. To discuss these phenomena in detail, molecular dynamics simulations of molecular chlorine are performed for three different state points. In general, the theory captures the behavior for small wavevector and frequencies as expected. For example, in the hydrodynamic regime and for molecular fluids with small moment of inertia like chlorine, the theory predicts that the longitudinal and transverse intrinsic angular velocity correlation functions are almost identical, which is also seen in the molecular dynamics simulations. However, the theory fails at large wavevector and frequencies. To account for the correlations at these scales, we derive a phenomenological expression for the frequency dependent rotational viscosity and wavevector and frequency dependent longitudinal spin viscosity. From this we observe a significant coupling enhancement between the molecular angular velocity and translational velocity for large frequencies in the gas phase; this is not observed for the supercritical fluid and liquid state points. () 2013 American Institute of Physics. [http://dx.doi.org/10.1063/1.4774095]
\end{abstract}

\section{INTRODUCTION}

It has been shown that for atomic fluids the Navier-Stokes equation correctly predicts the decay of the transverse velocity autocorrelation function for wavelengths down to two atomic diameters. ${ }^{1,2}$ For smaller wavelengths, the decay can be described phenomenologically by introducing a wavevector dependent viscosity, which is also frequency dependent and defined through the Navier-Stokes equation itself., ${ }^{1,3,4}$ This formalism can be termed generalized Navier-Stokes (GNS) theory since it involves a generalized Newtonian constitutive relation between the fluid stress and strain rate. Naturally, such a generalized theory can be extended to study other frequency and wavevector dependent transport phenomena such as heat conductivity and diffusion ${ }^{1,5}$ and this is now well established. ${ }^{3,6,7}$

The fact that there exists a coupling between the molecular intrinsic (or spin) angular momentum and the hydrodynamical degrees of freedom has long been known. ${ }^{8-13}$ Recently, the coupling has been shown to reduce the flow rate in highly confined geometries, ${ }^{14,15}$ that it is the mechanism behind flow generation when a rotating electrical field is applied across a channel, ${ }^{14,16}$ and that it can be utilized to perform plane wave pumping. ${ }^{17}$ The coupling is described by an extension of the Navier-Stokes (ENS) equations, ${ }^{9,10,13}$ in which

\footnotetext{
a) jschmidt@ruc.dk.
}

the dynamics of both the linear momentum density and the spin angular momentum density are given. Good agreement between the ENS equation and molecular dynamics simulation data has been found ${ }^{15,18}$ for fluids flowing in nano-slit pores.

The formulation of the ENS equations allows one to study additional multiscale correlation phenomena present in molecular fluids. The framework of such study is a natural extension of the GNS formalism and involves new correlation functions and wavevector and frequency dependent transport coefficients, not present in the classical theory. In this paper, we formulate the multiscale hydrodynamics as described by the ENS theory by deriving expressions for the wavevector dependent correlation functions, treating the transport coefficients as constants. These expressions are therefore only valid in the hydrodynamic regime, that is, for sufficiently small wavevectors and frequencies. Through the longitudinal autocorrelation function for the spin angular momentum it is shown that it is possible to define the frequency dependent rotational viscosity and the wavevector and frequency dependent longitudinal spin viscosity entering the ENS equations. We refer to this generalized formalism as generalized extended Navier-Stokes (GENS) theory. In principle, this theory can also be used to treat the frequency and wavevector dependent transverse spin viscosity, however, with some more effort. We also present molecular dynamics simulations of 
molecular chlorine at three different state points in order to discuss the multiscale correlation phenomena present in such fluids in the context of the theory.

The paper is organized as follows. In Sec. II, the GENS theory is presented. In Sec. III A, we give details about the molecular dynamics simulations and in Sec. III B we discuss the results from the simulations in the framework of theory. Section IV is devoted to a short summary and final remarks.

\section{FOUNDATION}

Our approach is founded in Onsager's regression hypothesis, ${ }^{19}$ which states that thermally induced fluctuations decay on average according to the deterministic equations of motion. Specifically, the decay of the correlations follows the deterministic description and is therefore dependent on the fluid transport properties. This approach also enables a direct comparison of the results with data obtained by equilibrium molecular dynamics simulations.

In the following, we consider a single component isotropic molecular fluid with a mass density $\rho$ composed of uniaxial molecules with moment of inertia per unit mass $I=\operatorname{tr}(\mathbf{I}) / 3$, where $\mathbf{I}$ is the moment of inertia tensor per unit mass. In the absence of an external force field and for sufficiently small pressure gradients, the convection term can be ignored and the linearized form of the ENS equations $\operatorname{read}^{13,20}$

$$
\begin{gathered}
\rho \frac{\partial \mathbf{u}}{\partial t}=-\nabla p+\left(\eta_{v}+\eta_{0} / 3-\eta_{r}\right) \nabla(\nabla \cdot \mathbf{u}) \\
+\left(\eta_{0}+\eta_{r}\right) \nabla^{2} \mathbf{u}+2 \eta_{r}(\nabla \times \boldsymbol{\Omega}), \\
\rho I \frac{\partial \boldsymbol{\Omega}}{\partial t}=2 \eta_{r}(\nabla \times \mathbf{u}-2 \boldsymbol{\Omega})+\left(\zeta_{v}+\zeta_{0} / 3-\zeta_{r}\right) \nabla(\nabla \cdot \boldsymbol{\Omega}) \\
+\left(\zeta_{0}+\zeta_{r}\right) \nabla^{2} \boldsymbol{\Omega},
\end{gathered}
$$

where $\mathbf{u}$ is the velocity field, $\boldsymbol{\Omega}$ the spin angular velocity field, and $\nabla p$ is the pressure gradient. Compared to the classical Navier-Stokes equation, the ENS equations involve additional transport coefficients. The transport coefficients $\eta_{v}, \eta_{0}$, and $\eta_{r}$ are the bulk, shear, and rotational viscosities, respectively, and $\zeta_{v}, \zeta_{0}$, and $\zeta_{r}$ the corresponding spin viscosities. ${ }^{13}$ It is worth noting that the dynamic equation for the spin angular velocity does not depend on the pressure gradient or temperature gradient, but only on the linear velocity gradient. ${ }^{13}$ In Fourier space, the ENS equations are given by

$$
\begin{gathered}
\rho \frac{\partial \widetilde{\mathbf{u}}}{\partial t}=-i \mathbf{k} \tilde{p}-\left(\eta_{v}+\eta_{0} / 3-\eta_{r}\right) \mathbf{k}(\mathbf{k} \cdot \widetilde{\mathbf{u}}) \\
-\left(\eta_{0}+\eta_{r}\right) k^{2} \widetilde{\mathbf{u}}+2 \eta_{r} i \mathbf{k} \times \widetilde{\boldsymbol{\Omega}} \\
\rho I \frac{\partial \widetilde{\boldsymbol{\Omega}}}{\partial t}=2 \eta_{r}(i \mathbf{k} \times \widetilde{\mathbf{u}}-2 \widetilde{\boldsymbol{\Omega}})-\left(\zeta_{v}+\zeta_{0} / 3-\zeta_{r}\right) \mathbf{k}(\mathbf{k} \cdot \widetilde{\boldsymbol{\Omega}}) \\
-\left(\zeta_{0}+\zeta_{r}\right) k^{2} \widetilde{\boldsymbol{\Omega}}
\end{gathered}
$$

where the Fourier transform is defined via

$$
\widetilde{f}(\mathbf{k}, t)=\int_{-\infty}^{\infty} f(\mathbf{r}, t) \exp (-i \mathbf{k} \cdot \mathbf{r}) \mathrm{d} \mathbf{r} .
$$

We let $\mathbf{k}=(0, k, 0)$ such that the components in the velocity and spin angular velocity fields can be written as purely transverse and longitudinal components. For the velocity field, we obtain

$$
\begin{gathered}
\rho \frac{\partial \tilde{u}_{x}}{\partial t}=-\left(\eta_{0}+\eta_{r}\right) k^{2} \widetilde{u}_{x}+2 \eta_{r} i k \widetilde{\Omega}_{z}, \\
\rho \frac{\partial \tilde{u}_{y}}{\partial t}=-i k \tilde{p}-\left(\eta_{v}+4 \eta_{0} / 3\right) k^{2} \widetilde{u}_{y}, \\
\rho \frac{\partial \tilde{u}_{z}}{\partial t}=-\left(\eta_{0}+\eta_{r}\right) k^{2} \tilde{u}_{z}-2 \eta_{r} i k \widetilde{\Omega}_{x},
\end{gathered}
$$

where $k^{2}=\mathbf{k} \cdot \mathbf{k}$. The rotational viscosity cancels in Eq. (4b), implying that the coupling has no effect in the longitudinal direction for the velocity. For the angular velocity field, the components are

$$
\begin{gathered}
\rho I \frac{\partial \widetilde{\Omega}_{x}}{\partial t}=-\left(4 \eta_{r}+\zeta k^{2}\right) \widetilde{\Omega}_{x}+2 \eta_{r} i k \tilde{u}_{z}, \\
\rho I \frac{\partial \widetilde{\Omega}_{y}}{\partial t}=-\left(4 \eta_{r}+\left(\zeta_{v}+4 \zeta_{0} / 3\right) k^{2}\right) \widetilde{\Omega}_{y}, \\
\rho I \frac{\partial \widetilde{\Omega}_{z}}{\partial t}=-\left(4 \eta_{r}+\zeta k^{2}\right) \widetilde{\Omega}_{z}-2 \eta_{r} i k \tilde{u}_{x} .
\end{gathered}
$$

For simplicity, we write

$$
\zeta=\zeta_{0}+\zeta_{r}
$$

where $\zeta$ is denoted the spin viscosity.

In the usual treatment, the dynamical equations are often Fourier-Laplace transformed with respect to time (yielding frequency dependence rather than time dependence), which leads to a system of linear algebraic equations of the form $\widetilde{\mathbf{x}}(\mathbf{k}, 0)=\mathbf{H}(\mathbf{k}, \omega) \cdot \widehat{\mathbf{x}}(\mathbf{k}, \omega)$, where $\widehat{\mathbf{x}}$ is the Fourier and Fourier-Laplace transform of the hydrodynamic field vector $\mathbf{x}$ (that includes density and energy/temperature), $\widetilde{\mathbf{x}}$ the Fourier transformed field at $t=0$, and $\mathbf{H}$ is the $5 \times 5$ hydrodynamic matrix. ${ }^{21}$ The transforms are defined in Eqs. (3) and (22). In the case of the ENS theory, the hydrodynamic field vector and matrix are readily redefined. If we write the field vector as $\mathbf{x}=\left(\rho, T, \mathbf{u}^{\prime}, \boldsymbol{\Omega}^{\prime}\right)$, where $T$ is the temperature, $\mathbf{u}^{\prime}$ and $\boldsymbol{\Omega}^{\prime}$ are permutations of the velocity and spin fields $\mathbf{u}^{\prime}=\left(u_{y}, u_{x}, u_{z}\right)$ and $\boldsymbol{\Omega}^{\prime}=\left(\Omega_{y}, \Omega_{x}, \Omega_{z}\right)$, the hydrodynamic matrix is an $8 \times 8$ matrix and can be written on a block form

$$
\mathbf{H}(\mathbf{k}, \omega)=\left[\begin{array}{cc}
\mathbf{H}_{1}^{N S}(\mathbf{k}, \omega) & \mathbf{0} \\
\mathbf{0} & \mathbf{H}_{2}^{E N S}(\mathbf{k}, \omega)
\end{array}\right]
$$

where $\mathbf{H}_{1}^{N S}:\left(\widehat{\rho}(\mathbf{k}, \omega), \widehat{T}(\mathbf{k}, \omega), \widehat{u}_{y}(\mathbf{k}, \omega)\right) \mapsto(\widetilde{\rho}(\mathbf{k}, t=0)$, $\left.\widetilde{T}(\mathbf{k}, t=0), \widetilde{u}_{y}(\mathbf{k}, t=0)\right)$ and has exactly the same entries as in the classical treatment, see Hansen and McDonald. ${ }^{21}$ The second block matrix $\mathbf{H}_{2}^{E N S}$ accounts for the remainder of the hydrodynamic field in the extended theory and is given by 


$$
\mathbf{H}_{2}^{E N S}=\left[\begin{array}{ccccc}
\eta_{t} k^{2} / \rho & 0 & 0 & 0 & -2 \eta_{r} i k / \rho \\
0 & \eta_{t} k^{2} / \rho & 0 & 2 \eta_{r} i k / \rho & 0 \\
0 & 0 & \left(4 \eta_{r}+\zeta_{l} k^{2}\right) / \rho I & 0 & 0 \\
0 & -2 \eta_{r} i k / \rho I & 0 & \left(4 \eta_{r}+\zeta k^{2}\right) / \rho I & 0 \\
2 \eta_{r} i k / \rho I & 0 & 0 & 0 & \left(4 \eta_{r}+\zeta k^{2}\right) / \rho I
\end{array}\right]+i \omega \mathbf{I},
$$

where

$$
\eta_{t}=\eta_{r}+\eta_{0} \text { and } \zeta_{l}=\zeta_{v}+4 \zeta_{0} / 3
$$

and $\mathbf{I}$ is the $5 \times 5$ unit matrix. We can of course proceed along this line; however, for the present purpose we will simply analyze Eqs. (4) and (5) and we just listed the hydrodynamic matrix here for completeness.

We first focus on the dynamics of the transverse velocity autocorrelation function (TVACF) and the transverse angular velocity autocorrelation function (TAVACF). Due to the system isotropy, we can choose between two equivalent pairs of transverse velocity and angular velocity fields; here, we simply choose the $\widetilde{u}_{x}$ and $\widetilde{\Omega}_{z}$ components (as they couple, see Eqs. (4) and (5)). From this, we define the (fluctuating) field vector $\widetilde{\mathbf{x}}(\mathbf{k}, t)=\left(\widetilde{u}_{x}, \widetilde{\Omega}_{z}\right)$ with $\langle\widetilde{\mathbf{x}}(\mathbf{k}, t)\rangle=\mathbf{0}$, and the corresponding correlation matrix

$$
\begin{aligned}
& \frac{1}{V}\langle\widetilde{\mathbf{x}}(\mathbf{k}, t) \widetilde{\mathbf{x}}(-\mathbf{k}, 0)\rangle \\
& \quad=\frac{1}{V}\left[\begin{array}{cc}
\left\langle\widetilde{u}_{x}(\mathbf{k}, t) \widetilde{u}_{x}(-\mathbf{k}, 0)\right\rangle & \left\langle\widetilde{u}_{x}(\mathbf{k}, t) \widetilde{\Omega}_{z}(-\mathbf{k}, 0)\right\rangle \\
\left\langle\widetilde{\Omega}_{z}(\mathbf{k}, t) \widetilde{u}_{x}(-\mathbf{k}, 0)\right\rangle & \left\langle\widetilde{\Omega}_{z}(\mathbf{k}, t) \widetilde{\Omega}_{z}(-\mathbf{k}, 0)\right\rangle
\end{array}\right],
\end{aligned}
$$

where $\langle\ldots\rangle$ denotes the ensemble average and $V$ is the system volume. The diagonal elements of this matrix are the TVACF and TAVACF, which we will write as

$$
\begin{gathered}
C_{u u}^{\perp}(\mathbf{k}, t)=\left\langle\widetilde{u}_{x}(\mathbf{k}, t) \widetilde{u}_{x}(-\mathbf{k}, 0)\right\rangle \frac{1}{V}, \\
C_{\Omega \Omega}^{\perp}(\mathbf{k}, t)=\left\langle\widetilde{\Omega}_{z}(\mathbf{k}, t) \widetilde{\Omega}_{z}(-\mathbf{k}, 0)\right\rangle \frac{1}{V} .
\end{gathered}
$$

According to Onsager's regression hypothesis, the dynamics of the correlation matrix is governed by the macroscopic equations and the solution is

$$
\langle\widetilde{\mathbf{x}}(\mathbf{k}, t) \widetilde{\mathbf{x}}(-\mathbf{k}, 0)\rangle=\boldsymbol{\Phi}(t) \cdot\langle\widetilde{\mathbf{x}}(\mathbf{k}, 0) \widetilde{\mathbf{x}}(-\mathbf{k}, 0)\rangle,
$$

where $\boldsymbol{\Phi}(t)$ is the fundamental matrix for the solution to the differential equation system Eqs. (4a) and (5c). Thus, we get

$$
\begin{gathered}
C_{u u}^{\perp}(\mathbf{k}, t)=\frac{k_{B} T}{\rho} \Phi_{u u}^{\perp}(t), \\
C_{\Omega \Omega}^{\perp}(\mathbf{k}, t)=\frac{k_{B} T}{2 I \rho} \Phi_{\Omega \Omega}^{\perp}(t),
\end{gathered}
$$

where the initial values follow from the equipartition theorem and

$$
\begin{aligned}
& \Phi_{u u}^{\perp}(t)=\left(\frac{1}{2}-A\right) \exp \left(-\lambda_{1} t\right)+\left(\frac{1}{2}+A\right) \exp \left(-\lambda_{2} t\right), \\
& \Phi_{\Omega \Omega}^{\perp}(t)=\left(\frac{1}{2}+A\right) \exp \left(-\lambda_{1} t\right)+\left(\frac{1}{2}-A\right) \exp \left(-\lambda_{2} t\right) .
\end{aligned}
$$

Here, the eigenvalues $\lambda_{1}$ and $\lambda_{2}$ are given by

$$
\begin{aligned}
\lambda_{1,2}= & \frac{1}{2 I \rho}\left[4 \eta_{r}+\left(\zeta+I \eta_{t}\right) k^{2}\right. \\
& \left. \pm \sqrt{16 \eta_{r}^{2}+8 \eta_{r}\left(\zeta+I\left(2 \eta_{r}-\eta_{t}\right)\right) k^{2}+\left(\zeta-I \eta_{t}\right)^{2} k^{4}}\right]
\end{aligned}
$$

and $A$ is

$$
A=\frac{4 \eta_{r}+\left(\zeta-I \eta_{t}\right) k^{2}}{2 I \rho\left(\lambda_{1}-\lambda_{2}\right)} .
$$

The off-diagonal elements in the correlation matrix, $C_{u \Omega}^{\perp}(\mathbf{k}, t)$ and $C_{\Omega u}^{\perp}(\mathbf{k}, t)$, do not vanish. This can be understood by evaluating the real and imaginary parts of the cross correlation functions separately under phase space coordinate inversion. Here, the real part must vanish, but not the imaginary part. It can also be seen directly from the fundamental matrix where

$$
\begin{gathered}
\Phi_{u \Omega}^{\perp}(t)=-\frac{i 2 k \eta_{r}}{\rho\left(\lambda_{1}-\lambda_{2}\right)}\left[\exp \left(-\lambda_{1} t\right)-\exp \left(-\lambda_{2} t\right)\right], \\
\Phi_{\Omega u}^{\perp}(t)=\frac{i 2 k \eta_{r}}{\rho I\left(\lambda_{1}-\lambda_{2}\right)}\left[\exp \left(-\lambda_{1} t\right)-\exp \left(-\lambda_{2} t\right)\right] .
\end{gathered}
$$

We will later show that this result is in qualitative agreement with molecular dynamics data.

The longitudinal angular velocity autocorrelation function (LAVACF), $C_{\Omega \Omega}^{\|}(\mathbf{k}, t)$, is defined as

$$
C_{\Omega \Omega}^{\|}(\mathbf{k}, t)=\left\langle\widetilde{\Omega}_{y}(\mathbf{k}, t) \widetilde{\Omega}_{y}(-\mathbf{k}, 0)\right\rangle \frac{1}{V},
$$

and is predicted from Eq. (5b) to decay as a simple single exponential function

$$
C_{\Omega \Omega}^{\|}(\mathbf{k}, t)=\frac{1}{2 I \rho} \exp \left[\frac{-\left(4 \eta_{r}+\zeta_{l} k^{2}\right)}{\rho I} t\right] .
$$

The rotational viscosity $\eta_{r}$ can be found from this correlation function. To see this, we first form the dynamic equation for 
$C_{\Omega \Omega}^{\|}(\mathbf{k}, t),{ }^{22}$ i.e., multiplying Eq. (5b) with $\widetilde{\Omega}_{y}(-\mathbf{k}, 0)$ and ensemble averaging gives

$$
\rho I \frac{\partial C_{\Omega \Omega}^{\|}}{\partial t}=-\left(4 \eta_{r}+\zeta_{l} k^{2}\right) C_{\Omega \Omega}^{\|}
$$

Fourier-Laplace transforming and rearranging, we get

$$
\chi_{\Omega \Omega}^{\|}(\mathbf{k}, \omega)=\rho I \frac{C_{\Omega \Omega}^{\|}(\mathbf{k}, 0)-i \omega \widehat{C}_{\Omega \Omega}^{\|}(\mathbf{k}, \omega)}{\widehat{C}_{\Omega \Omega}^{\|}(\mathbf{k}, \omega)},
$$

where

$$
\widehat{C}_{\Omega \Omega}^{\|}(\mathbf{k}, \omega)=\int_{0}^{\infty} C_{\Omega \Omega}^{\|}(\mathbf{k}, t) \exp (-i \omega t) \mathrm{d} t
$$

and where we will assume that $\chi_{\Omega \Omega}^{\|}$can be decomposed into two terms

$$
\chi_{\Omega \Omega}^{\|}(\mathbf{k}, \omega)=4 \eta_{r}(\omega)+\zeta_{l}(\mathbf{k}, \omega) k^{2} .
$$

Note that the longitudinal spin viscosity, $\zeta_{l}$, is now wavevector and frequency dependent, whereas we conjecture that the rotational viscosity is only frequency dependent since it describes a local relaxation process. This assumption may not be strictly valid, but as we will show later it yields a correct value for the rotational viscosity in the zero frequency limit and can account for the observed dynamics of the correlation functions. The frequency dependent rotational viscosity is thus given by

$$
\eta_{r}(\omega)=\frac{1}{4} \lim _{\mathbf{k} \rightarrow \mathbf{0}} \chi_{\Omega \Omega}^{\|}(\mathbf{k}, \omega),
$$

which provides a formal method to calculate the rotational viscosity directly from the LAVACF. The response function $\chi_{\Omega \Omega}^{\|}$also enables one to calculate the longitudinal spin viscosity kernel, i.e., rearranging Eq. (23) we get

$$
\zeta_{l}(\mathbf{k}, \omega)=\frac{\chi_{\Omega \Omega}^{\|}(\mathbf{k}, \omega)-4 \eta_{r}(\omega)}{k^{2}}=\frac{\Delta \chi_{\Omega \Omega}^{\|}(\mathbf{k}, \omega)}{k^{2}},
$$

with $k^{2} \neq 0$ and where $\Delta \chi_{\Omega \Omega}^{\|}(\mathbf{k}, \omega) \equiv \chi_{\Omega \Omega}^{\|}(\mathbf{k}, \omega)$ $-\lim _{\mathbf{k} \rightarrow \mathbf{0}} \chi_{\Omega \Omega}^{\|}(\mathbf{k}, \omega)$. A phenomenological expression for the frequency and wavevector dependent spin viscosity $\zeta(\mathbf{k}, \omega)$ is not readily found due to the solution to the TAVACF. However, in the limit of small wavevector and inertia an approximate expression can be obtained, as we will see below.

\section{A. Limit of small inertia and wavevector}

In the hydrodynamic limit, one can expand Eq. (15) in terms of $k$ giving

$$
\lambda_{1}=\frac{4 \eta_{r}+\left(I \eta_{r}+\zeta\right) k^{2}}{\rho I}+O\left(k^{4}\right), \quad \lambda_{2}=\frac{\eta_{0} k^{2}}{\rho}+O\left(k^{4}\right)
$$

and

$$
A=\frac{1}{2}+\frac{1}{4} I k^{2}+O\left(k^{4}\right) \text {. }
$$

For small moment of inertia, $I$, this means that $\lambda_{1} \approx\left(4 \eta_{r}+\zeta k^{2}\right) /(\rho I)$ and $A \approx 1 / 2$, and this yields the

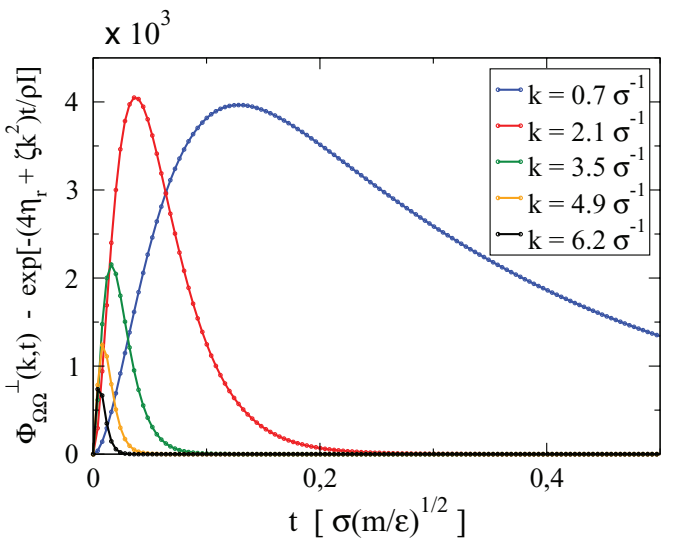

FIG. 1. Liquid chlorine. Comparison between Eq. (13b) and the approximation given by Eq. (28b) for different wavevectors. The units are reduced molecular dynamics units, $\sigma=3.3321 \AA, \epsilon=2.46210 \times 10^{-21} \mathrm{~J}$ and $m=35.45 \mathrm{~g} / \mathrm{mol}$.

approximate solutions to the TVACF and TAVACF

$$
\begin{aligned}
C_{u u}^{\perp}(\mathbf{k}, t) & =\frac{k_{B} T}{\rho} \exp \left[-\frac{\eta_{0} k^{2}}{\rho} t\right], \quad(k \rightarrow 0), \\
C_{\Omega \Omega}^{\perp}(\mathbf{k}, t) & =\frac{k_{B} T}{2 I \rho} \exp \left[-\frac{\left(4 \eta_{r}+\zeta k^{2}\right)}{\rho I} t\right], \quad(k \rightarrow 0) .
\end{aligned}
$$

The approximation to the TAVACF is compared with the full solution for liquid chlorine in Fig. 1; the transport coefficients are listed Table I. It is clearly seen that the absolute difference is very small even for large wavevectors. The reason why there exists a maximum is that both $\Phi_{\Omega \Omega}^{\perp}$ and $\exp \left[-\left(4 \eta_{r}+\zeta k^{2}\right) / \rho I\right]$ have value 1 at $t=0$, and the difference decreases for larger times since both predictions rapidly decay to zero here. Naturally, the difference increases for increasing moment of inertia.

Equation (28b) has the same form as Eq. (19), hence, in the strict limit $k=0$ the LAVACF and TAVACFs are identical and an equivalent expression of Eq. (24) can be given in terms of the TAVACF.

Interestingly, Eq. (28a) recaptures the result from the classical Navier-Stokes theory, where the coupling is ignored, but where no truncation with respect to wavevector is done. One can think of the classical approach as an atomic picture,

TABLE I. Values of the relevant transport coefficients in the limit of zero frequency and wavevector. $\eta_{0}$ and $\zeta$ are evaluated using a Green-Kubo integral, ${ }^{13}$ whereas $\eta_{r}$ is calculated from Eqs. (24) and (36), see text for details. The standard errors associated with $\eta_{r}$ (third column) in the gas and fluid phases are in the order of $10^{-5}$ and not given in the table. The value of $\eta_{r}$ in the liquid state is in excellent agreement with previously published results by Moore et al. ${ }^{24}$ and Delhommelle. ${ }^{35}$

\begin{tabular}{lcccc}
\hline \hline State point & $\eta_{0}$ & $\eta_{r}$ [Eq. (36)] & $\eta_{r}$ [Eq. (24)] & $\zeta$ \\
\hline Gas & $0.17 \pm 0.02$ & 0.0012 & $0.0019 \pm 0.0006$ & $0.0106 \pm 0.0004$ \\
Supercrit. & $0.71 \pm 0.08$ & 0.037 & $0.041 \pm 0.005$ & $0.056 \pm 0.001$ \\
fluid & & & & \\
Liquid & $6.71 \pm 0.55$ & $0.42 \pm 0.03$ & $0.44 \pm 0.04$ & $0.69 \pm 0.02$ \\
\hline
\end{tabular}


and we write this result ${ }^{1,21}$

$$
C_{u u}^{A, \perp}(\mathbf{k}, t)=\frac{k_{B} T}{\rho} \exp \left[-\frac{\eta_{0} k^{2}}{\rho} t\right],
$$

where we use superscript $A$ to indicate that this is what would be obtained in an atomic description (also of a molecular fluid), see Ref. 23 for a discussion. The reason for the difference between Eqs. (13a) and (29) lies in that the ENS equations are based on a description where the molecular pressure tensor is allowed to have an anti-symmetric part, ${ }^{10}$ and where an additional balance equation for the internal molecular degrees of freedom is introduced. As seen above, the difference between these two descriptions vanishes in the hydrodynamic limit, as one would expect.

\section{MOLECULAR DYNAMICS OF MOLECULAR CHLORINE}

\section{A. Simulation details}

The correlation functions can be evaluated using molecular dynamics simulations. We will here study molecular chlorine at three different state points, corresponding to a dilute fluid/gas, a supercritical fluid, and a liquid. The chlorine molecule is modelled as in Ref. 24, by letting the two chlorine atoms be bonded via a flexible bond. The total potential energy in the system is then

$$
U=\sum_{\text {pairs }} U_{L J}\left(r_{i j}\right)+\sum_{\text {bonds }} U_{B}\left(r_{i j}\right),
$$

where $r_{i j}$ is the distance between chlorine atoms $i$ and $j$. The pair potential energy, $U_{L J}$, is given by the truncated and shifted Lennard-Jones potential ${ }^{22}$

$$
U_{L J}\left(r_{i j}\right)=4 \epsilon\left(\left(\sigma / r_{i j}\right)^{12}-\left(\sigma / r_{i j}\right)^{6}\right)-U_{L J}^{C} \text { if } r_{i j} \leq r_{c},
$$

where $\epsilon$ and $\sigma$ are characteristic energy and length scales, respectively, $U_{L J}^{C}$ the value of the unshifted potential at the truncation, $r_{c}=2.5 \sigma$. If $r_{i j}>r_{c}, U_{L J}=0$. We will, as is common practice and unless otherwise stated, express mechanical quantities in units of $\epsilon, \sigma$ and the atomic mass $m$. For example, the temperature is $T^{*}=k_{B} T / \epsilon$, number density is $n^{*}=n \sigma^{3}$ and so forth. ${ }^{25}$ Furthermore, we shall also not write the asterisk for convenience. If one wishes to translate the reduced units into real units $\sigma=3.3321 \AA, \epsilon=2.46210 \times 10^{-21} \mathrm{~J}$ and $m=35.45 \mathrm{~g} / \mathrm{mol}$, see Ref. 26 and references therein. The pair interaction only acts between non-bonded atoms. The bond potential function is given by a harmonic spring potential

$$
U_{B}\left(r_{i j}\right)=\frac{1}{2} k_{s}\left(r_{i j}-l_{B}\right)^{2} .
$$

Here, $k_{s}=2500$ is the spring constant and $l_{B}=0.63$ is the zero-force bond length. It has been shown that this spring constant keeps the average bond length close to $l_{B}$ with vibrations that are still slow enough to be accurately integrated forward in time with the time step used, $h=0.001 .^{24}$

Initially, 1200 molecules are arranged on a simple lattice with mass density 0.05 . The simulation box is then compressed with relatively high compression rate in the $x$ and $z$ directions and small rate in the $y$ direction. This gives a rect- angular geometry with dimensions $L_{x} \times L_{y} \times L_{z}$, where $L_{x}$ $=L_{z}$. The geometry allows one to study the system for small wavevectors $\mathbf{k}=(0, k, 0)$, where $k=2 \pi n_{k} / L_{y}, n_{k}=1,2, \ldots$, 25. The different state points studied are: $(T, \rho)=(0.98$, $1.088),(4.0,0.5),(2.0,0.1)$, corresponding to liquid, supercritical fluid, and gas state points. Because chlorine is a diatomic molecule the molecular number density $n$ is simply half the mass density. The equations of motion are integrated using the leap-frog algorithm. ${ }^{27}$ The system is coupled to a Nosé-Hoover thermostat ${ }^{28,29}$ to simulate the canonical (NVT) ensemble.

After compression and equilibration the wavevector dependent transverse velocity is found from the definition of the momentum density $\mathbf{j}(\mathbf{r}, t)=\rho \mathbf{u}(\mathbf{r}, t)$ assuming that we can ignore the effect of density fluctuations, i.e., the microscopic variable is $\mathbf{u}(\mathbf{r}, t)=(1 / \rho) \sum_{i} M_{i} \mathbf{v}_{i}(t) \delta\left(\mathbf{r}-\mathbf{r}_{i}\right)$, where $M_{i}$ is the mass of molecule $i, \mathbf{v}_{i}$ is the center-of-mass velocity, and $\mathbf{r}_{i}$ is the center-of-mass position vector of molecule $i$. Here, we only study a single component liquid, and so the $x$-component of the transverse velocity field is

$$
\tilde{u}_{x}(\mathbf{k}, t)=\frac{1}{n} \sum_{i=1}^{N} v_{x, i}(t) \exp \left(-i k y_{i}(t)\right),
$$

where $N$ is the number of molecules in the simulation system, $v_{x, i}$ is the $x$-component velocity, and $y_{i}$ is its $y$ component center-of-mass. For isotropic fluids composed of uniaxial molecules, the relation between the microscopic angular velocity and the angular velocity is $I \Omega(\mathbf{r}, t)$ $=\left(I_{p} V / N\right) \sum_{i} \Omega_{i}(t) \delta\left(\mathbf{r}-\mathbf{r}_{i}\right),{ }^{30-32}$ where $I_{p}$ is the principal moment of inertia per unit mass. The transverse spin angular velocity is thus given by

$$
\widetilde{\Omega}_{z}(\mathbf{k}, t)=\frac{3}{2 n} \sum_{i=1}^{N} \Omega_{z, i}(t) \exp \left(-i k y_{i}(t)\right),
$$

where $\Omega_{z, i}$ is the spin angular velocity of molecule $i$ and where we have used the fact that $I=2 I_{p} / 3$ for uniaxial molecules. The spin angular velocity is calculated from the angular momentum $s_{z, i}$ via $\Omega_{z, i}=s_{z, i} / I_{p}$ with $I_{p}=0.099$. Likewise, the longitudinal spin angular field is

$$
\widetilde{\Omega}_{y}(\mathbf{k}, t)=\frac{3}{2 n} \sum_{i=1}^{N} \Omega_{y, i}(t) \exp \left(-i k y_{i}(t)\right) .
$$

From the definitions (33)-(35), the TVACF, TAVACF, and LAVACF are then readily computed.

The relevant transport coefficients, $\eta_{0}, \eta_{r}$, and $\zeta$ are also evaluated in the zero wavevector and zero frequency limit. To this end we calculate the molecular pressure tensor and the couple tensor from which the coefficients can be found; we refer the reader to Refs. 13, 33, and 34 for details. The results are listed in Table I. One important point to note here is that the rotational viscosity does not have a Green-Kubo integral and its evaluation involves an assumption about its Laplace transform. ${ }^{33}$ It has been shown ${ }^{6,14,24,33}$ that the following simple Lorentzian form is a good approximation

$$
\eta_{r}(s)=\frac{\eta_{r}(0)}{1+s \tau}
$$



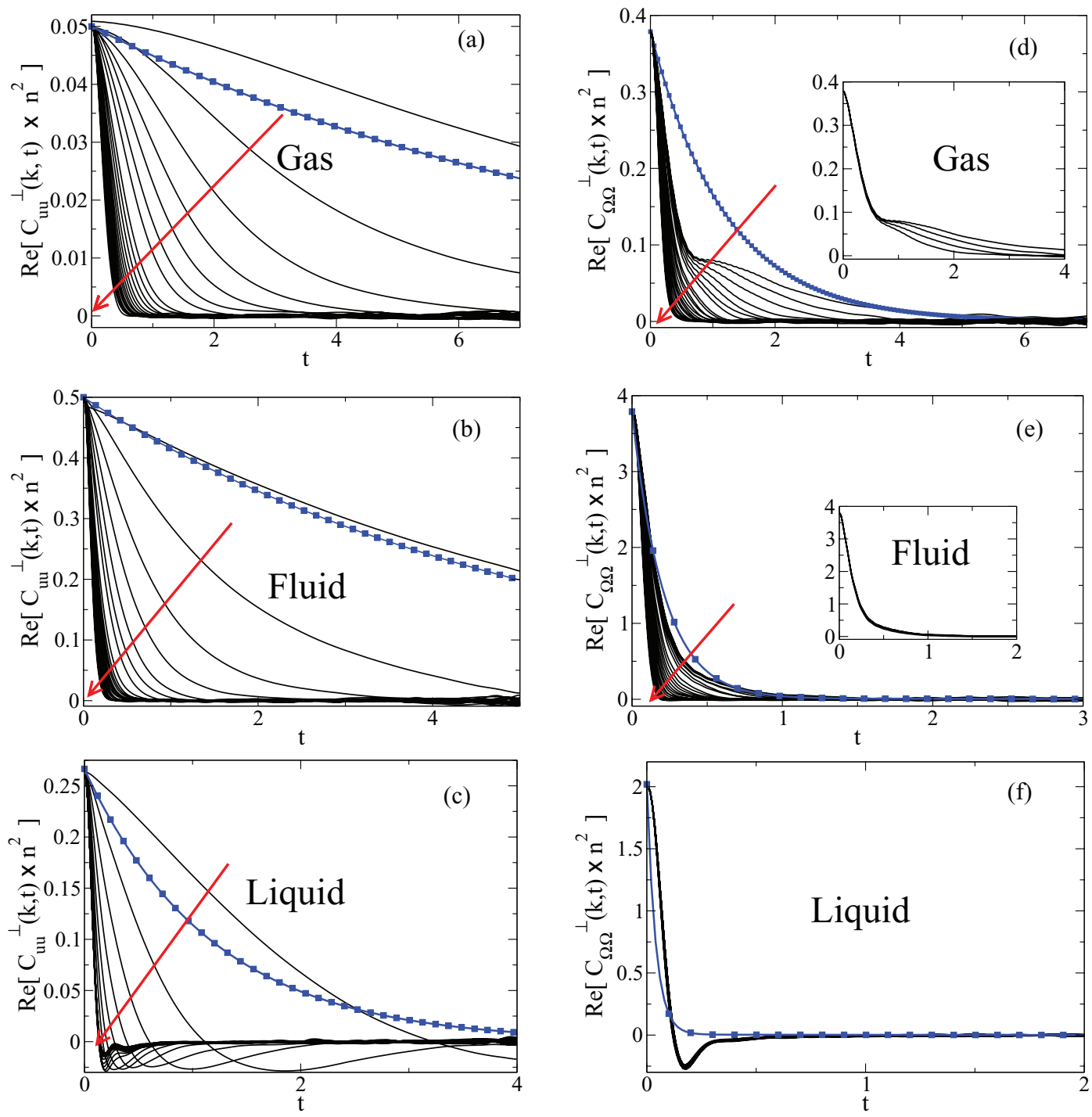

FIG. 2. TVACFs and TAVACFs multiplied with the squared number density for molecular chlorine at the three different state points and for small times. For the gas state point $0.25<k<6.25$, the fluid state point $0.36<k<8.30$ and for the liquid state point $0.37<k<8.62 . t$ is in units of 1.9 ps. The insets in (d) and (e) show the TAVACFs for the smallest four wavevectors. Arrows indicate direction of increasing wavevector. Blue filled squares connected with lines are the predictions from the theory for the lowest wavevector.

where $s$ is the complex Laplace variable, $\eta_{r}(0)=\eta_{r}$ is the zero-frequency rotational viscosity, and $\tau$ is a characteristic relaxation time. This functional form was first suggested by Evans and Hanley. ${ }^{33}$ The procedure involves fitting to Eq. (36), but the resulting rotational viscosity depends somewhat on the range of $s$ chosen. While the method is satisfactory, it is still desirable to devise a first-principle procedure for evaluating the rotational viscosity without assuming a functional form for $\eta_{r}$ valid over a range of $s$. Equation (24) shows that this is possible in the framework of GENS theory.

\section{B. Simulation results}

Figure 2 shows the TVACFs and TAVACFs for a range of wavevectors for all three state points. First, it is noted that for sufficiently small wavevectors the TVACFs decay, to a good approximation, as a single exponential function for the gas and fluid state points. This is in agreement with the ap- proximation, Eq. (28a). Fits of the data for low wavevector to Eq. (28a) yield shear viscosities of $\eta_{0}=0.17 \pm 0.03$ and $\eta_{0}=0.64 \pm 0.05$ for the gas and fluid phases, respectively. These are within statistical uncertainty the same values obtained in the strictly hydrodynamic regime, see Table I. This means that the decay of the TVACF is correctly predicted by the approximative solution and therefore also the classical Navier-Stokes equation. This is true even for wavelengths down to around $5.5 \mathrm{~nm}$ for the gas and supercritical fluid phases.

For large wavevectors, the decay of the TVACF no longer follows a single exponential. A Prony series analysis reveals that the decay cannot be described satisfactorily as a sum of two exponentials, as predicted by Eqs. (13a) and (14a). In particular, this is true for the liquid state point Fig. 2(c). This discrepancy between theory and simulation data is linked to the short time and spatial correlations present in the fluid, motivating the introduction of wavevector and frequency dependent transport coefficients. ${ }^{4}$ 

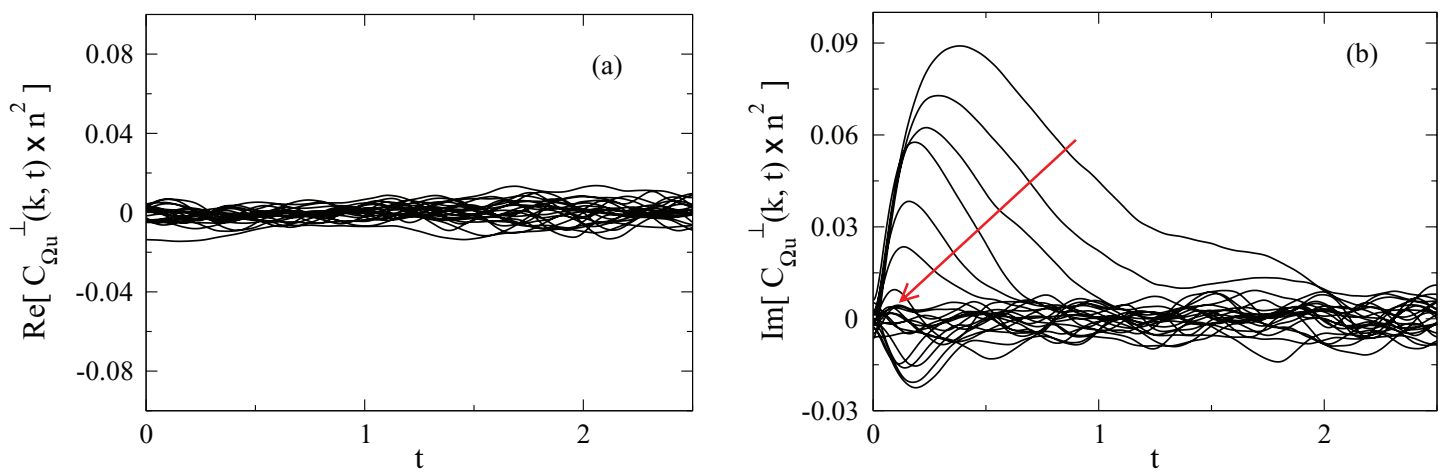

FIG. 3. Cross correlation function $C_{\Omega u}(\mathbf{k}, t)$ at the fluid state point. Arrows indicate direction of increasing wavevector.

The TAVACF for the gas state is shown in Fig. 2(d). The decay for low wavevectors is characterized by two distinct regimes (or modes) (i) a wavevector independent fast regime and (ii) a wavevector dependent slow regime. This behavior is in qualitative agreement with Eqs. (13b) and (14b), but due to the values of the transport coefficient the theory cannot account for the distinct regimes. Therefore, in the limit of small wavevector, we associate the fast regime with the relaxation of spin angular momentum since this is a local non-diffusive process. This interpretation supports the conjecture that the rotational viscosity is wavevector independent as proposed in Sec. II. Also, we interpret the decay rate for very short times to be due to the frequency dependent rotational viscosity, i.e., the rotational viscosity increases for increasing frequency in this region. The wavevector dependent behavior at large times is a fingerprint of the spin diffusion process, and is captured reasonably well by Eqs. (13b), (14b), and Eq. (28b). For large wavevectors, the diffusive mode dominates the decay as expected from the theory.

In Fig. 2(f), the TAVACF for liquid chlorine is shown. The anti-correlation for small times highlights the "rattling" of the molecules. From Table I, we see that the rotational and spin viscosities are of similar magnitude at zero wavevector, but it is striking that in the liquid phase, the effect of the spin diffusion is not observed for any of the wavelengths studied here. This indicates that, for the liquid, the spin viscosity quickly goes to zero for increasing wavevectors. As discussed previously, in the hydrodynamic regime the effect of spin dif-

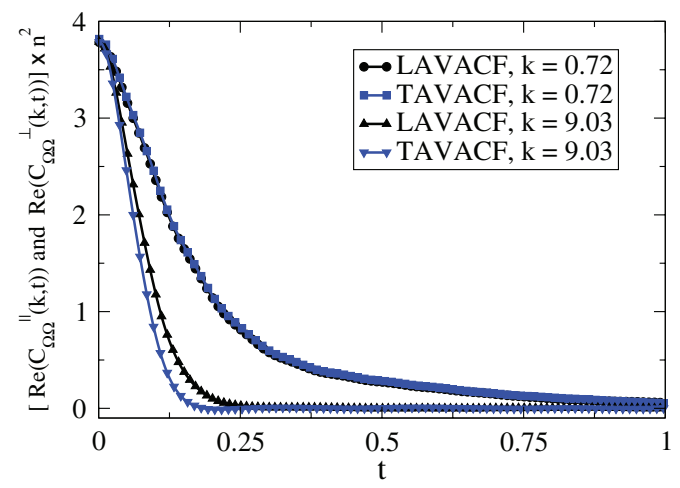

FIG. 4. Comparison between the LAVACF and the TAVACF for the fluid state point. fusion is negligible, meaning that it has no effect on the decay of the TAVACF.

In Sec. II, it was seen that the real part of the cross correlation function is zero, whereas the imaginary part was nonzero. This is confirmed in the molecular dynamics simulations, Fig. 3 where the real part, (a), and imaginary part, (b), are plotted for the fluid state point.

In the hydrodynamic limit and for small moment of inertia, the LAVACF and TAVACF should have the approximate same decay, see Eqs. (19) and (28b). Indeed this is also observed in the simulations data. In fact, for the gas and liquid state points the TAVACF and LAVACFs were indistinguishable within statistical error for all wavevectors studied here. Only for the fluid state point a small, but significant difference was observed for high values of $k$; see Fig. (4). This strongly indicates that $\zeta_{l}(\mathbf{k}, \omega) \approx \zeta(\mathbf{k}, \omega)$.

Table I lists the rotational viscosity found from Eq. (24) in the limit of zero frequency. Good agreement with the value obtained by using the Evans and Hanley method ${ }^{33}$ is observed. In addition, the real part of the frequency dependent rotational viscosity is shown in Fig. 5. It is worth noting that $\eta_{r}$ has a peak in the gas phase, which supports the assumption above that $\eta_{r}(\omega)$ increases at this state point for large frequencies. This also means that the coupling between the molecular spin and the fluid velocity is increasing for larger frequencies

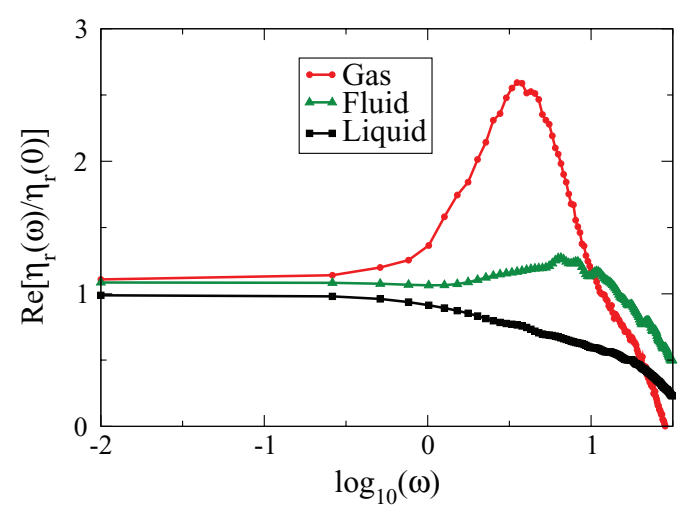

FIG. 5. Real part of the rotational viscosity for the three different state points for $\omega>0.01$ and relative to the zero frequency value, $\eta_{r}(0)$, given in Table I column three. Before performing the Fourier-Laplace transform all data were averaged and a Hann window ${ }^{36}$ was applied to the resulting single data set in order to obtain the best possible curves. 


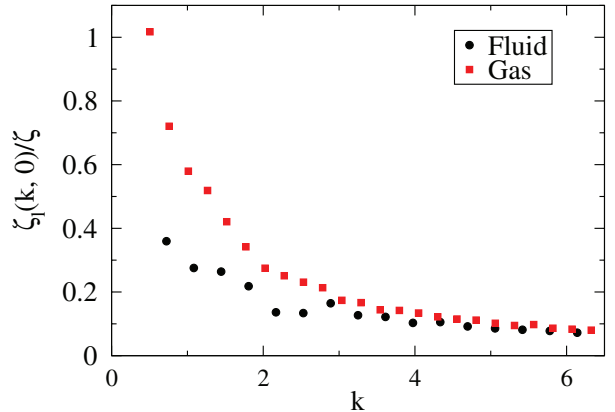

FIG. 6. Zero frequency longitudinal spin viscosity as a function of wavevector relative to $\zeta$ given in Table I. The kernel for the liquid state point is zero for all wave vectors shown here. As in Fig. 5 the data were averaged and a Hann window ${ }^{36}$ was applied before Fourier-Laplace transforming.

in the case of a dilute system. This clearly significant coupling enhancement is not observed for the other state points.

Due to statistical noise in the simulations $\Delta \chi_{\Omega \Omega}^{\|}$approaches zero slower than $k^{2}$, see Eq. (25), within statistical uncertainty, i.e., the expression for the wavevector and frequency dependent spin viscosity cannot be resolved properly as $k^{2} \rightarrow 0$. This means in practice that the zero wavevector longitudinal spin viscosity, $\zeta_{l}$, cannot be evaluated using Eq. (25). However, the expression can be applied here for non-zero wavevectors and illustrates the points made above. Figure 6 shows the wavevector dependence of the longitudinal spin viscosity in the limit of zero frequency. For the liquid state point the spin viscosity is zero for $k>0.75$, corresponding to a wavelength of approximately $0.5 \mathrm{~nm}$. This is a direct consequence of the decay of the TAVACF, which is governed by the wavevector independent spin relaxation mechanism. On the other hand, for the two dilute systems it is clearly seen that the spin diffusion significantly affects the decay of the LAVACF for large wavevectors. We stress again that this result is specifically for chlorine and the conclusion cannot be generalized to other molecular liquids. Another important point is that the longitudinal spin viscosity kernel appears to approach the spin viscosity $\zeta$ at low wavevectors indicating once again that $\zeta \approx \zeta_{l}$.

\section{SUMMARY}

In this paper, we have formulated the generalized extended Navier-Stokes theory that includes the coupling between the spin angular momentum and linear momentum. This enabled an expression for the rotational viscosity and longitudinal spin viscosity kernels under the assumption that the former response function is wavevector independent. For molecular fluids where the moment of inertia is very small and in the hydrodynamic limit the theory can be simplified significantly, and it was shown that the longitudinal and transverse angular velocity correlation functions feature approximately the same decay.

The theoretical predictions were tested by molecular dynamics simulations of molecular chlorine. In general, the theory captures the overall behavior for small wavevector and frequencies as expected. However, it does not correctly account for the detailed relaxation dynamics at small length and time scales as expected. From the molecular dynamics simulations, we evaluated the longitudinal spin viscosity and rotational viscosity kernels. From this, we noticed a coupling enhancement at high frequencies at the gas state point.

\section{ACKNOWLEDGMENTS}

J.S.H. wishes to acknowledge Lundbeckfonden for supporting this work as part Grant No. R49-A5634. The centre for viscous liquid dynamics "Glass and Time" is sponsored by the Danish National Research Foundation (DNRF). Also, the authors are grateful for the very useful reviewer comments.

${ }^{1}$ W. E. Alley and B. J. Alder, "Generalized transport coefficients for hard spheres," Phys. Rev. A 27, 3158 (1983).

${ }^{2}$ J. S. Hansen, P. J. Daivis, K. P. Travis, and B. D. Todd, "Parameterization of the nonlocal viscosity kernel for an atomic fluid," Phys. Rev. E 76, 041121 (2007).

${ }^{3}$ J. P. Boon and S. Yip, Molecular Hydrodynamics (Dover, New York, 1991).

${ }^{4}$ B. J. Palmer, "Transverse-current autocorrelation-function calculations of the shear viscosity for molecular liquids," Phys. Rev. E 49, 359 (1994).

${ }^{5}$ T. Hunt and B. D. Todd, "Diffusion of linear polymer melts in shear and elongational flows," J. Chem. Phys. 131, 054904 (2009).

${ }^{6}$ D. Bertolini and A. Tani, "Stress tensor and viscosity of water: Molecular dynamics and generalized hydrodynamics results," Phys. Rev. E 52, 1699 (1995).

${ }^{7}$ J. M. O. de Zárate and J. V. Sengers, Hydrodynamic Fluctuations (Elsevier, Amsterdam, 2006).

${ }^{8}$ M. Born, "The mobility of electrolyte ions," Z. Phys. 1, 221 (1920).

${ }^{9} \mathrm{H}$. Grad, "Statistical mechanics, thermodynamics and fluid dynamics of systems with an arbitrary number of integrals," Commun. Pure Appl. Math. 5, 455 (1952).

${ }^{10}$ S. R. de Groot and P. Mazur, Non-equilibrium Thermodynamics (Dover, 1984).

${ }^{11}$ R. F. Snider and K. S. Lewchuk, "Irreversible thermodynamics of a fluid system with spin," J. Chem. Phys. 46, 3163 (1967).

${ }^{12}$ N. K. Ailawadi, B. J. Berne, and D. Forster, "Hydrodynamics and collective angular-momentum fluctuations in molecular fluids," Phys. Rev. A 3, 1462-1472 (1971).

${ }^{13}$ D. J. Evans and W. B. Streett, "Transport properties of homonuclear diatomics. II. Dense fluids," Mol. Phys. 36, 161-176 (1978).

${ }^{14}$ J. S. Hansen, H. Bruus, B. D. Todd, and Peter J. Daivis, "Rotational and spin viscosities of water: Application to nanofluidics," J. Chem. Phys. 133, 144906 (2010).

${ }^{15}$ J. S. Hansen, J. C. Dyre, P. J. Daivis, B. D. Todd, and H. Bruus, "Nanoflow hydrodynamics," Phys. Rev. E 84, 036311 (2011).

${ }^{16}$ J. D. Bonthuis, D. Horinek, L. Bocquet, and R. R. Netz, "Electrohydraulic power conversion in planar nanochannels," Phys. Rev. Lett. 103, 144503 (2009).

${ }^{17}$ B. U. Felderhof, "Efficiency of magnetic plane wave pumping of a ferrofluid through a planar duct," Phys. Fluids 23, 092003 (2011).

${ }^{18}$ J. Delhommelle and D. J. Evans, "Poiseuille flow of a micropolar fluid," Mol. Phys. 100, 2857-2865 (2002).

${ }^{19}$ L. Onsager, "Reciprocal relations in irreversible processes. I," Phys. Rev. 37, 405 (1931).

${ }^{20}$ D. W. Condiff and J. S. Dahler, "Fluid mechanical aspects of antisymmetric stress," Phys. Fluid 6, 842 (1964).

${ }^{21}$ J. P. Hansen and I. R. McDonald, Theory of Simple Liquids (Academic, London, 1986).

${ }^{22}$ D. A. Mcquarrie, Statistical Mechanics (Harper, New York, 1976).

${ }^{23}$ B. D. Todd and P. J. Daivis, "Homogeneous non-equilibrium molecular dynamics simulations of viscous flow: techniques and applications," Mol. Simul. 33, 189 (2007).

${ }^{24}$ R. J. D. Moore, J. S. Hansen, and B. D. Todd, "Rotational viscosity of linear molecules: an equilibrium molecular dynamics study," J. Phys. Chem. 128, 224507 (2008).

${ }^{25}$ M. P. Allen and D. J. Tildesley, Computer Simulation of Liquids (Clarendon, New York, 1989). 
${ }^{26}$ M. L. Matin, P. J. Daivis, and B. D. Todd, "Comparison of planar shear flow and planar elongational flow for systems of small molecules," J. Chem. Phys. 113, 9122-9131 (2000).

${ }^{27}$ D. Frenkel and B. Smit, Understanding Molecular Simulation (Academic, London, 1996)

${ }^{28} \mathrm{~S}$. Nosé, "A molecular dynamics method for simulation in the canonical ensemble," Mol. Phys. 52, 255-268 (1984).

${ }^{29}$ W. G. Hoover, "Canonical dynamics: Equilibrium phase-space distributions," Phys. Rev. A 31, 1695-1697 (1985).

${ }^{30}$ S. Sarman and D. J. Evans, "Statistical mechanics of viscous flow in nematic fluids," J. Chem. Phys. 99, 9021-9036 (1993).

${ }^{31}$ K. P. Travis, B. D. Todd, and D. J. Evans, "Poiseuille flow of molecular fluids," Physica A 240, 315 (1997).
${ }^{32}$ K. P. Travis and D. J. Evans, "Molecular spin in a fluid undergoing Poiseuille flow," Phys. Rev. E 55, 1566 (1997).

${ }^{33}$ D. J. Evans and H. J. M. Hanley, "Fluctuation expressions for fast thermal processes: Vortex viscosity," Phys. Rev. A. 25, 1771-1774 (1982).

${ }^{34}$ J. S. Hansen, P. J. Daivis, and B. D. Todd, "Viscous properties of isotropic fluids composed of linear molecules: Departure from the classical NavierStokes theory in nanoconfined geometries," Phys. Rev. E 80, 046322 (2009).

${ }^{35}$ J. Delhommelle, "Rotational viscosity of uniaxial molecules," Mol. Phys. 100, 3479-3482 (2002).

${ }^{36}$ W. H. Press, W. T. Vettering, S. A. Teukolsky, and B. P. Flannery, Numerical Recipes in C (Cambridge University Press, Cambridge, 1992). 